\title{
USING THE AE METHOD TO INCREASE THE LIFETIME OF COMPLEX-SHAPED STEEL PARTS
}

\author{
A. L. Bobrov \\ Siberian transport university, 191 D. Kovalchuk st., 630049, Novosibirsk, Russian Federation \\ *Corresponding author. E-mail: beaver@stu.ru; address for correspondence: ul. D. Kovalchuk 191, 630049, \\ Novosibirsk, Russian Federation; Tel.:+7 3833280 346; Fax: +7 3833280346
}

The author has developed way to extend the life of cast components of freight car bogies using acoustic emission method. The proposed modified integral method of the determination state of the cast parts truck freight wagons in the parameters of acoustic emission signals the most loaded sections. Present a study at developing criteria for assessing the state of the cast parts and the results of the application on real details.

Keywords: acoustical emission, criteria for evaluation, cast details, parameter, lifetime.

DOI: $10.17804 / 2410-9908.2016 .2 .021-033$

\section{References}

1. Smirnov A.N., Gerike B.L., Muraviev V.V. Diagnostirovanie tekhnicheskikh ustroistv opasnykh proizvodstvennykh obiektov [Diagnostics of Hazardous Industrial Facilities]. Novosibirsk, Nauka Publ., 2003, 244 p. (In Russian).

2. Kollakot R. Diagnostika povrezhdeniy [Diagnostics of Damages]. M., Mir Publ., 1989, 516 p. (In Russian).

3. Ivanov V.I. Estimation of Extra Useful Life of Objects by Monitoring Acoustic-Emission Evaluation of additional time operation of using acoustic emission monitoring. Soviet Journal of Nondestructive Testing-USSR, 1982, vol. 18, iss. 11, pp. 891-894.

4. $\quad$ Bobrov A.L. A method of predicting residual life of metal products, RF Patent 2361199.

5. Bobrov A.L., Stepanova L.N., Kabanov S.I., Lebedev E.Yu. Method of forecasting the residual resource of metal products and device for its implementation, RF Patent 2448343.

6. Abramov O.V., Gradov O.M. Identification of some processes of evolution of imperfect material structure with sources of spontaneous acoustic signals. Kontrol. Diagnostika, 2000, no. 6, pp. 15-21. (In Russian).

7. Berman A.V., Berman D.V., Artemenko A.I., Shakhmeister Yu.L., Vorontsova I.A., Berman O.A., Shteintsaig R.M., Khaspekov P.R. A method for predicting the residual life of steelworks, RF Patent 2193772. (In Russian).

8. Robsman V.A. Nonlinear transformation of acoustic emission probability distributions with the evolution of the defect ensemble in a solid. Acoustical Physics, 1996, vol. 42, no. 6, pp. 749-754.

9. Stepanova L.N., Bobrov A.L., Kanifadin K.V., Chernova V.V. Studying the principal parameters of acoustic emission signals in the static and cyclic testing of 20GL steel specimen. Deformatsiya i razrushenie, 2014, no. 6, pp. 41-47. (In Russian).

10. Nedoseka A.Ya., Nedoseka S.A. Acoustic emission and the lifetime of structures. Tekhnicheskaya diagnostika i nerazrushayushchiy kontrol, 2008, no. 2, pp. 5-19. (In Russian).

11. Grassmann S.A., Bolchanov A.A., Stepanova L.N., Bekher S.A., Bobrov A.L., Kabanov S.I. Acoustic Emission Testing of a freight bogie solebar. Zheleznodorozhnyy transport, 2011, no. 12, pp. 45-47. (In Russian).

12. Stepanova L.N., Grassmann S.A., Bobrov A.L., Bekher S.A., Bolchanov A.A. Acoustic emission testing of solebars with boxlike cross section. Russian Journal of Nondestructive testing, 2011, vol. 47, iss. 3, pp. 158-163. DOI: 10.1134/S1061830911030090. 
13. PB 03-593-03. Pravila organizatsii i provedeniya akustiko-emissionnogo kontrolya sosudov, apparatov, kotlov i tekhnichnologicheskikh truboprovodov [Rules for Organizing and Performing Acoustic Emission Testing of Vessels, Apparatuses, Boilers and Industrial Pipelines], M., GUP Nauchno-tekhnicheskiy tsentr po bezopasnosti i promyshlennosti. Gostekhnadzor Rossii, 2003, 56 p. (In Russian).

14. Bobrov A.L. Analysys of Variations of the Dynamic Characteristics of Acoustic Emission Sources under Static Loading of Metal Speciemens. Russian Journal of Nondestructive Testing, 2009, vol. 45, iss. 5, pp. 304-309. DOI: 10.1134/S1061830909050027. 
Подана в журнал: 24.12 .2015

УДК 620.179

DOI: $10.17804 / 2410-9908.2016 .2 .021-033$

\title{
ИСПОЛЬЗОВАНИЕ АЭ МЕТОДА ПРИ ПРОДЛЕНИИ СРОКА СЛУЖБЫ СТАЛЬНЫХ ДЕТАЛЕЙ СЛОЖНОЙ КОНСТРУКЦИИ
}

\author{
А. Л. Бобров \\ Сибирский государственный университет путей сообщения, 630049, г. Новосибирск, \\ ул. Дуси Ковальчук, 191, СГУПС \\ *Ответственный автор. Электронная почта: beaver@stu.ru; адрес для переписки: 630049, \\ г. Новосибирск, ул. Дуси Ковальчук, 191. Телефон: +7 (383) 328-03-46; факс: +7 (383) 328-03-46
}

В статье рассмотрен способ продления срока службы литых деталей тележек грузовых вагонов методом акустической эмиссии. Предлагается модифицированный интегральный метод оценки состояния деталей на основании параметров сигналов акустической эмиссии наиболее нагруженных участков. Приводятся исследования, направленные на выработку критериев оценки состояния литых деталей и результаты применения на реальных изделиях.

Ключевые слова: акустическая эмиссия, критерий оценки, литые детали, параметры, срок службы.

\section{1. Введение}

Современные методы НК позволяют решать диагностические задачи с высокой надежностью для объектов достаточно простой формы, имеющих однородную структуру и свойства. Вместе с тем существует ряд объектов, конструктивно сложных, неоднородных по структуре и имеющих внутренние допустимые дефекты, - литые металлические изделия для транспорта и тяжелого машиностроения. Наличие сложной формы и, как следствие, сильной неравномерности напряженного состояния в процессе эксплуатации, большой спектр литейных дефектов для большинства методов НК не позволяют с необходимой достоверностью оценивать текущее состояние и тем более остаточный ресурс таких объектов. Кроме того, подобного рода объекты (сложная форма, неоднородность структуры, неравномерное по времени изменение напряженно-деформированного состояния в разных локальных участках объекта, изменение геометрических размеров в процессе эксплуатации вследствие износов) являются наиболее обобщенным случаем в практике НК и оценки ресурса.

Достоверное определение ресурса технических объектов одна из важнейших задач, успешное решение которой позволяет существенно экономить капитальные вложения производственных и транспортных предприятий. Как правило, эта задача решается комплексом методов [1,2], в том числе с применением метода АЭ [2-5].

Последнее десятилетие исследования, связанные с определением долговечности материалов АЭ методом, ведутся в трех основных направлениях:

- $\quad$ определение связей структурного состояния различных материалов с параметрами АЭ и их физическое обоснование;

- $\quad$ анализ акустических волн, излучаемых развивающимися усталостными дефектами (прежде всего трещинами);

- $\quad$ модели практического применения метода АЭ для определения ресурса реальных объектов.

При исследованиях АЭ, генерируемой структурными изменениями материала, интересный подход зависимости скорости работы источника дислокаций и частоты столкновений движущихся дислокаций, скорости их отрыва и неподвижными барьерами (участками накопления и развития макродефектов) от характеристик неискаженных сигналов АЭ приво- 
дится в работе [6]. При этом метод имеет ряд существенных ограничений, связанных с решением задачи восстановления первичного (в момент генерирования) сигнала АЭ. Если решение такой задачи для изотропных объектов простой формы принципиально возможно, то существенные случайные искажения акустического тракта в объекте сложной формы учесть нельзя, и это существенно усложняет задачу.

Другой подход к решению проблемы приводит в качестве критерия оценки структурного состояния, прогнозирования прочностных свойств и долговечности квазихрупкого материала параметр, связанный с отношением энергии АЭ за определенный промежуток времени механических испытаний к суммарной энергии диссипации за этот же период [7]. Этот критерий слабо зависит от временного интервала испытаний, но жестко связан с относительным напряжением разрушения. Несмотря на то, что исследования касались керамических материалов, несомненно, что для металлов хотя бы частично действуют те же механизмы генерации АЭ при механическом статическом нагружении. Основными трудностями в этом подходе являются точное определение энергии диссипации и то, что нагружение следует проводить в неупругой области. Это всегда существенно сказывается на надежности ОК при последующем использовании.

Обобщая исследования в области определения структурно-напряженного состояния металлов методом АЭ, можно сделать следующие выводы:

- $\quad$ метод может определять характерные структурные состояния (например, переход от упругого деформирования к пластическому, состояние предразрушения), соответствующие точкам бифуркации в процессе эволюции напряженного состояния [8];

- при испытаниях в областях напряжений, соответствующих рабочим, т. е. существенно ниже напряжения упругопластического перехода, наиболее полную картину структурно-напряженного состояния дает циклическое нагружение, и чем больше число циклов, тем более объективные результаты получаются [9];

- $\quad$ в условиях производства уровень характеристик потока будет существенно зависеть от уровня шумов, не связанных с АЭ материала, и как следствие, искажать результаты диагностики [10].

\section{2. Постановка задачи}

Основные задачи системы оценки безотказной работы для детали сложной формы с неравномерно распределенным напряженным состоянием:

1) поиск потенциально опасных локальных участков;

2) ранжирование обнаруженных потенциально опасных участков по степени развития дефектов на них;

3) оценка срока безотказной эксплуатации на основании полученных сведений об объекте.

Высокой достоверности диагностики можно достичь при наличии информации о дефектности деталей. Поэтому в случае ответственных деталей подвижного состава применена комплексная методика, которая определяет наличие макроскопических дефектов и величину нагрузки на диагностируемый участок, а также степень их активности при приложении нагрузки, превышающей рабочую динамическую на 25 \%.

Методика АЭ диагностирования на конкретный срок эксплуатации была предложена в работе [4]. Параметры нагружения, аппаратура и настраиваемые характеристики изложены в работе [11]. Приемные преобразователи были установлены в соответсвии с рис. 1.

Суть методики заключается в том, что исходя из статистических данных, вся деталь разбивается на участки различной степени потенциальной опасности. В нашем случае мы опирались на исследования, приведенные во второй главе, и исходили из двух параметров: доли данного участка, бракуемой по наличию усталостной трещины, и оценки скорости роста трещины. Боковая рама и надрессорная балка были разбиты на участки, приведенные на 
рис. 2. Они подразделяются на четыре категории: самым темным цветом (1) обозначены наиболее опасные, для которых критерии оценки самые жесткие, это внутренние углы буксового проема боковой рамы, бурты наклонных плоскостей и средняя часть нижнего пояса надрессорной балки. Остальные по мере убывания опасности окрашены в более светлосерый цвет $(2,3)$. Основной объем металла цветом не выделен и к нему наименьшие требования.

При контроле деталь подвергается нагрузке, на 20-25 \% превышающей динамическую, возникающую в литых деталях в процессе эксплуатации. При этом приемные преобразователи фиксируют сигналы и аналитическим способом диагностическая система определяет координаты источников. Затем из общего массива сигналов выделяются источники в виде локальных участков, в которых сосредоточены акты АЭ с расстоянием между местоположением актов меньше погрешности измерений. Для каждого источника определяется число сигналов $N_{x}$ и дополнительные энергетические параметры. Оценка проводится по принципу, заложенному в интегральном методе оценки [12].
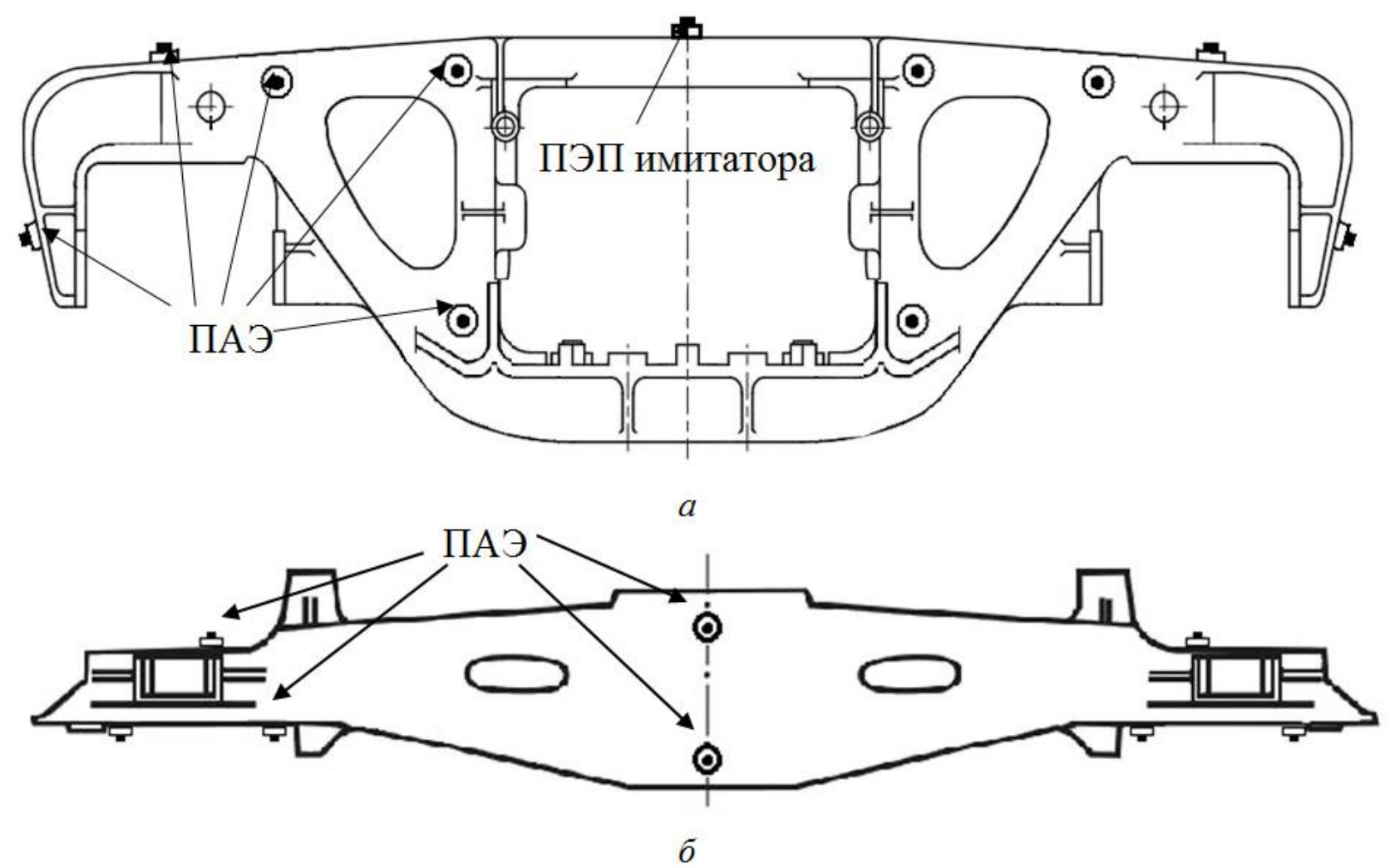

Рис. 1. Схема установки приемных преобразователей на боковой раме $(a)$ и надрессорной балке (б) 

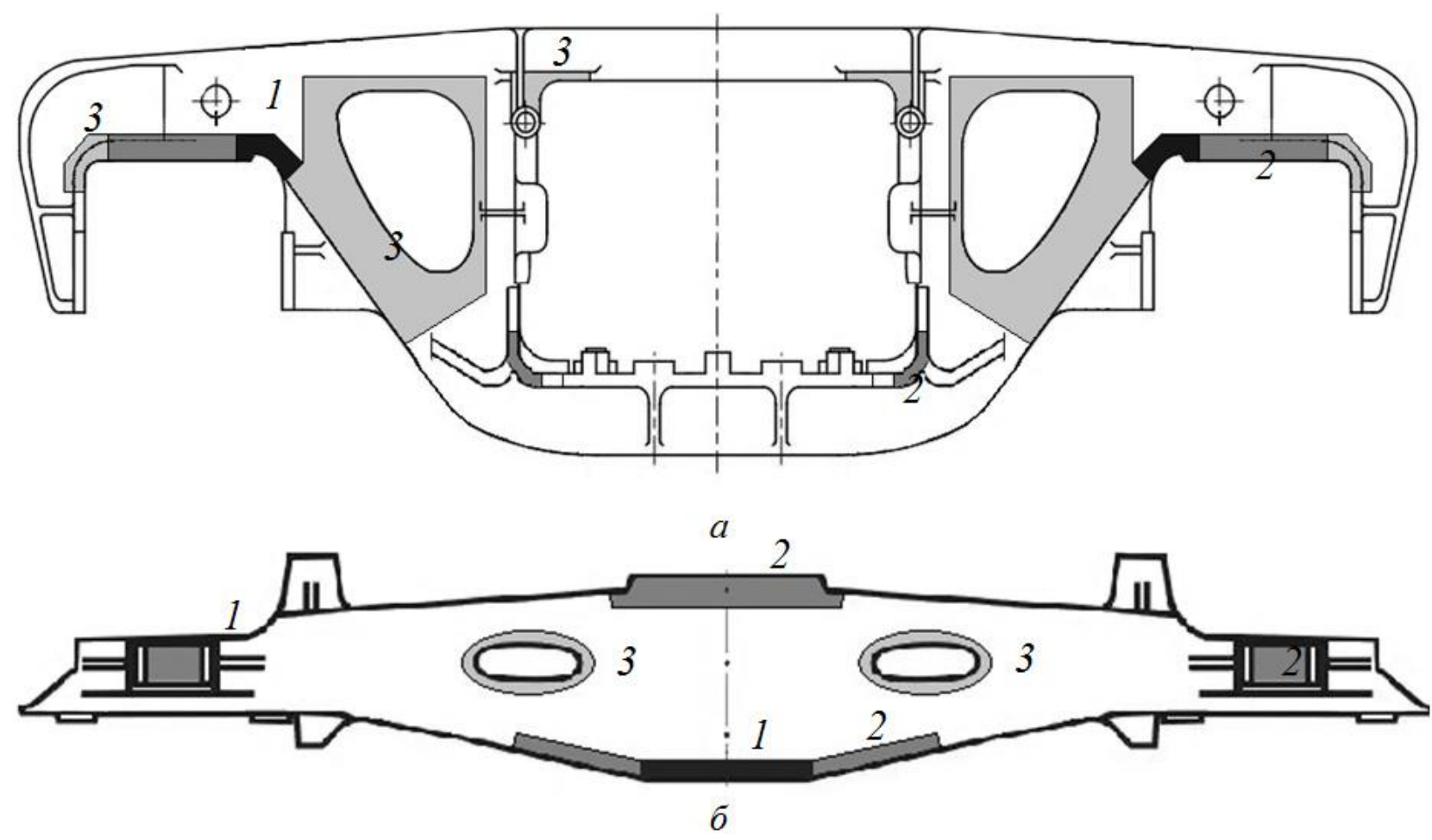

Рис. 2. Участки контроля на боковой раме $(a)$ и надрессорной балке (б)

\section{3. Результаты и обсуждение}

При определении критериев оценки использовали исследования образцов и результаты контроля деталей с развивающимися трещинами. Как показали результаты, наиболее надежным параметром может служить суммарная энергия сигналов АЭ из локального источника, а также коэффициент корреляции между сигналами источника. Для диагностирования деталей с целью продления срока эксплуатации использовали среднее значение коэффициента корреляции. Кроме того, при нагружении контролируемых деталей нагрузкой, превышающей на 20-25 \% рабочую, необходимо измерять напряжения или нагрузку для определения весового коэффициента для энергии сигналов. Таким образом, добиваемся слабого влияния энергии сигналов АЭ, принятых при малых нагрузках.

Для определения энергетического параметра воспользуемся исследованиями образцов с учетом затухания ультразвуковых волн в материале. Для этого применены данные исследования АЭ при статических испытаниях образцов с трещинами различных размеров. Анализировали данные образца, который не имел внутренних дефектов литья на пути трещины. При обработке результатов нагружения образцов с концентраторами получаем зависимость, приведенную на рис. 3. 


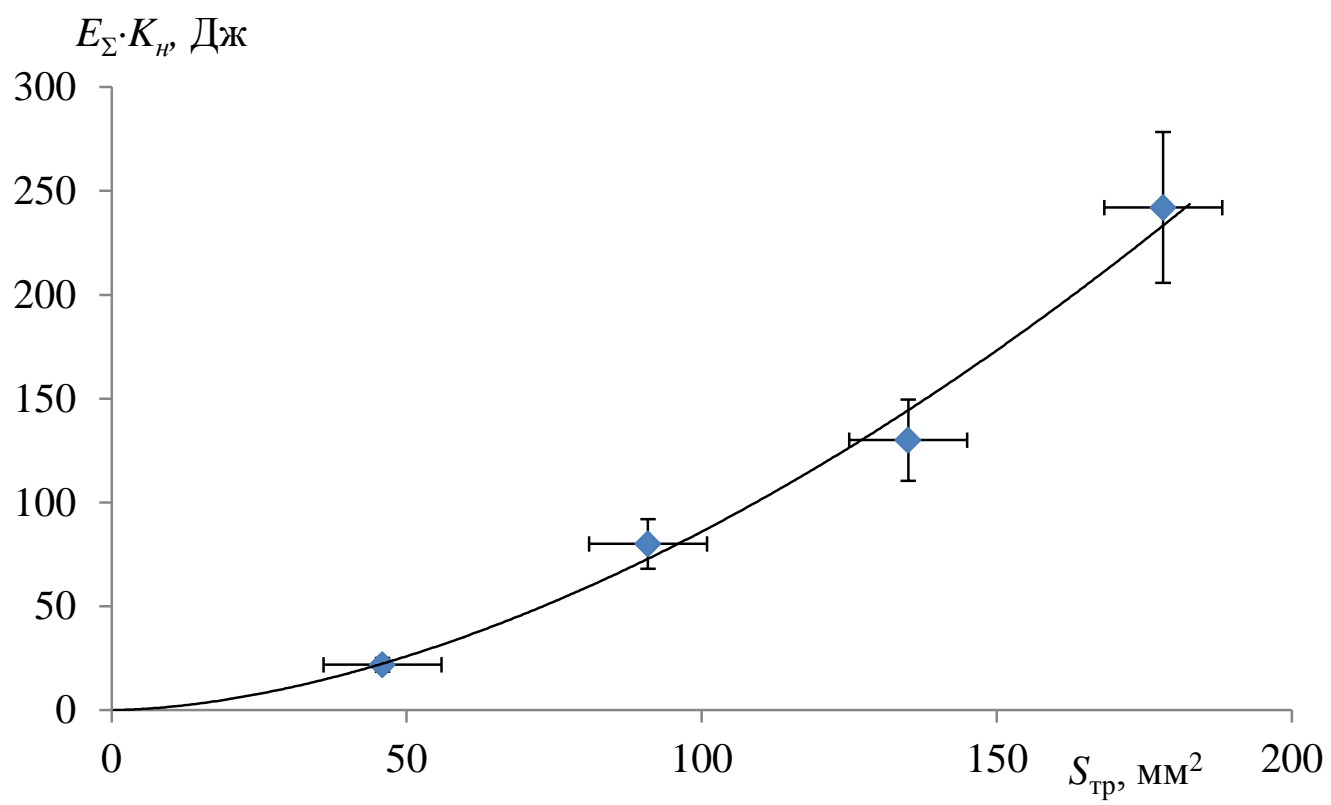

Рис. 3. График зависимости суммарной энергии сигналов АЭ с учетом коэффициента нагрузки от площади развития трещины

Такое взвешенное значение суммарной энергии $E_{\Sigma k}$ определяется формулой:

$$
E_{\Sigma k}=\sum_{i=1}^{n} E_{i} \cdot \frac{P_{i}}{P_{\max }}
$$

где $E_{i}$ - энергия $i$-го сигнала $P_{i}$ и $P_{\max }$ - соответственно нагрузка при приеме $i$-го сигнала и максимальная в испытаниях; $n$ - число сигналов акустической эмиссии в источнике.

А связь такой суммарной энергии $E_{\Sigma k}$ с площадью развивающейся трещины выражается экспериментально определенной степенной зависимостью:

$$
E_{\Sigma k}=0,03 \cdot S_{\mathrm{Tp}}^{1,73}
$$

Используя формулу (2), можно определить, что при развитии трещины даже на 1 мм² $^{2}$ возможна регистрация сигнала от источника с амплитудой, превышающей порог срабатывания системы на 15..17 дБ, если чувствительность соответствует той, которая была при исследовании образцов, и расстояние до источника будет аналогичным. Эти значения достижимы и вполне реализуемы, в том числе в деповских условиях, поэтому в дальнейшем будем считать чувствительность аналогичной или приводить ее к этому значению.

Однако штатными методами контроля выявляются плоские дефекты типа трещин площадью не менее 5 мм² $^{2}$ к тому же ими невозможно определить развивается дефект или нет. Как показывают результаты внеплановых отцепок вагонов, такой чувствительности для наиболее опасных зон 1 недостаточно, так как в этих зонах трещины могут развиться до разрушения в полноценный межремонтный период, поэтому в зонах 1 энергетический параметр должен быть минимально возможным (рис. 3). В связи с этим для зон 1 энергетический параметр примем равным $0,1 \mathrm{~B}^{2}$. В зонах 2 и 3 значение энергетического параметра будет соответственно 0,3 и $0,5 \mathrm{~B}^{2}$. В остальных частях детали энергетический параметр принимаем равным 1, что соответствует площади развивающейся трещины 7,2 мм². Однако это предельное состояние $E_{\min }$, которое рассчитано на максимальный срок эксплуатации деталей. Если же срок эксплуатации детали будет сокращен, то для энергетического критерия возможно повышение значения. 
Для повышения надежности идентификации источника используем корреляционный параметр, который позволяет определять стадию развития источника и минимизировать ложные источники, не связанные с развитием дефекта [13]. Корреляционный критерий применим в привязке к энергии тех сигналов, коэффициент взаимной корреляции для которых будем определять, кроме того, дополнительно следует учитывать и весовой коэффициент нагрузки. Таким образом, корреляционный параметр определим по формуле:

$$
Q_{i j}=\sum_{\substack{i=1 \\ j=2 \\ i \neq j}}\left\{\left(\frac{E_{i}+E_{j}}{2}\right) \cdot \frac{P_{j}}{P_{\max }} \cdot k_{i j}\right\},
$$

где $Q_{i j}$ - интегральная характеристика корреляции $x$-го источника сигнала акустической эмиссии в $y$-й зоне детали для двух соседних по времени прихода сигналов; $k_{i j}-$ коэффициент корреляции между сигналами акустической эмиссии $i$ и $j ; P_{j}-$ нагрузка во время регистрации сигнала при большей нагрузке из пары.

В процессе ресурсных испытаний интегральные критерии определяются для всех зафиксированных источников АЭ, а о степени поврежденности объекта и, следовательно, о возможности и времени его дальнейшей эксплуатации судят по дефекту, имеющему наибольшие интегральные характеристики (табл. 1).

Таблица 1 - Категории опасности источников АЭ в зависимости от полученных значений интегральных характеристик

\begin{tabular}{|l|l|l|l|}
\hline$Q_{i j}, E_{\Sigma k}$, Дж & $E_{\Sigma k}<E_{\min }$ & $E_{\min }<E_{\Sigma k}<E_{\max }$ & $E_{\Sigma k}>E_{\max }$ \\
\hline$Q_{i j}<Q_{\min }$ & Незначительный & Незначительный & Малозначительный \\
\hline$Q_{\min }<Q_{i j}<Q_{\max }$ & Малозначительный & Значительный & Значительный \\
\hline$Q_{i j}>Q_{\max }$ & Значительный & Критический & Критический \\
\hline
\end{tabular}

Для повышения надежности этот способ рекомендуется использовать с измерением напряженно-деформированного состояния деталей с помощью тензометрирования, но, как показала дальнейшая практика, он вполне работоспособен и при использовании других подтверждающих наличие дефектов методов НК, например магнитных методов дефектоскопии локальных участков, на которых обнаружен источник.

Для такого подхода к определению срока эксплуатации деталей следует определить не только максимальное недопустимое значение энергетического параметра, но и значение $E_{\max }$ - максимальное пороговое, а также аналогичные значения для корреляционного критерия и смысл качественных определений источников (табл. 1).

С учетом классификации дефектов будем исходить из того, что чем опаснее дефект, тем короче срок эксплуатации объекта. Статистические исследования подтверждают, что трещины малых размеров не всегда являются развивающимися и АЭ контроль при обеспечении должного уровня чувствительности способен адекватно разделить развивающиеся и неразвивающиеся трещины. Кроме того, если активный дефект, зарегистрированный АЭ методом, имеет параметры менее максимальных, значит, он развивается медленнее в силу отсутствия на пути литейных дефектов структуры или пониженном напряженном состоянии в силу, например, большей толщины стенок в конкретной контролируемой детали. Как показывают статистические исследования разрушений деталей в наиболее опасных участках, все они развивались в течение достаточно длительного времени эксплуатации. На рис. 4 пред- 
ставлена гистограмма распределения частоты изломов по километражу $w$, который был пройден вагоном с разрушенной деталью от последнего освидетельствования, когда дефекта еще не было. Следует отметить, что нормативный километраж между деповскими ремонтами составляет 160 тыс. км, за исключением дополнительных условий.

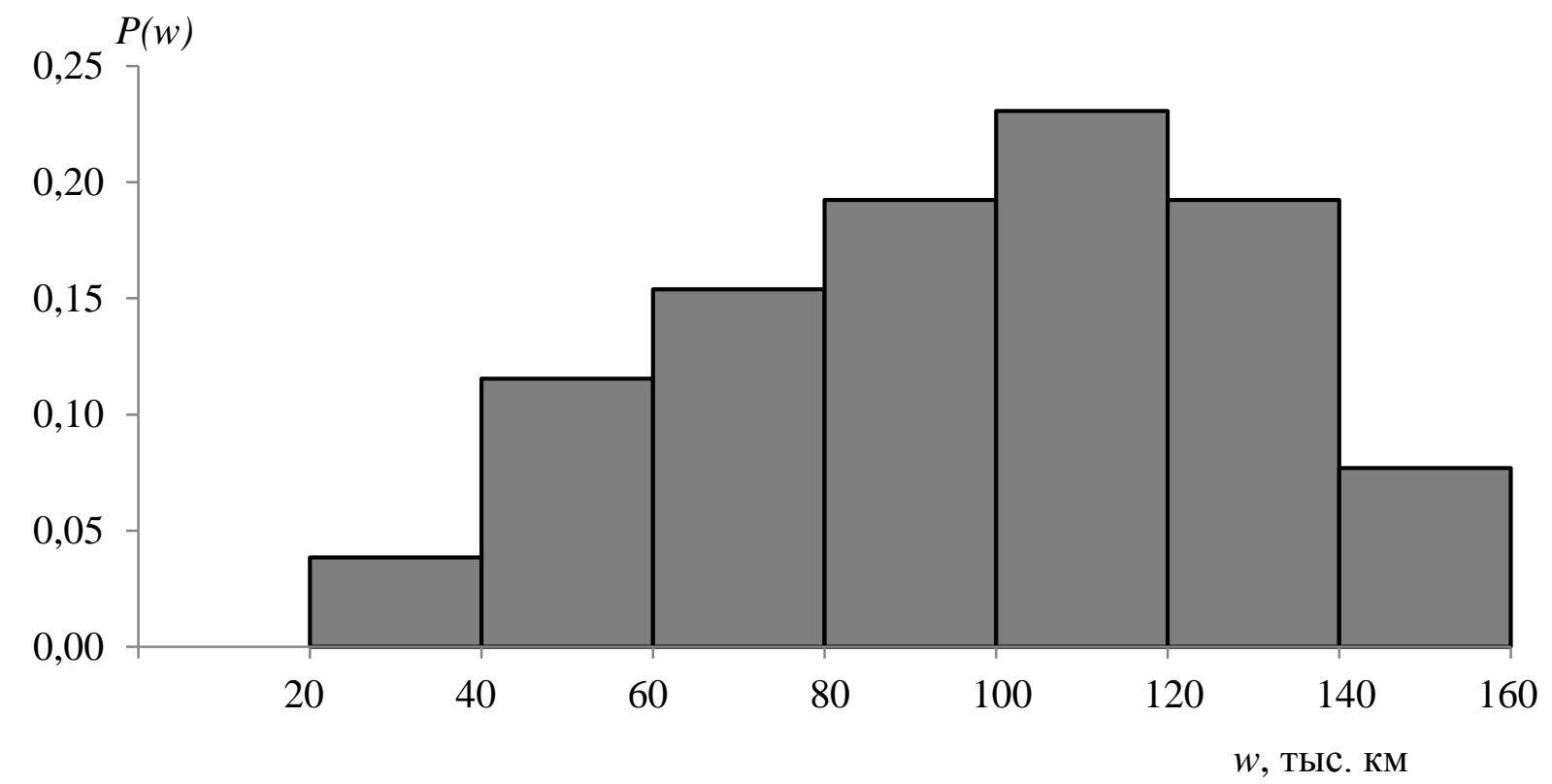

Рис. 4. Распределение частоты разрушения боковой рамы по развивающейся трещине во внутреннем углу буксового проема в зависимости от пробега вагона от последней диагностики

Полученные данные показывают, что скрытые дефекты малых размеров развиваются в среднем дольше, чем половина времени межремонтного пробега, при котором осуществляется НК деталей. Из этого следует, что повысив пороговые значения критериев оценки литых деталей до значений, эквивалентных обнаруживаемым магнитными методами, можно снизить срок гарантированной эксплуатации в два раза. При этом количество разрушений может быть снижено минимум на 70 \%. Приведенные на рис. 4 распределения показывают, что возможно принципиальное разбиение межремонтного срока эксплуатации при продлении ресурса детали на доли, что в ряде случаев экономически удобно для собственников деталей, при этом вероятность разрушения будет низкой. В нашем случае АЭ диагностирования состояния литых деталей тележек грузовых вагонов разобьем межремонтный пробег вагонов на доли 0,$25 ; 0,5$ и 0,75 , соответствующие значительным, малозначительным и незначительным источникам сигналов АЭ.

Далее установим пороговые значения критериев оценки $E_{\min } ; E_{\max } ; Q_{\min } ; Q_{\max }$ на основании тех же экспериментальных данных, которые использовали для предельного энергетического критерия. Дополнительно проведены испытания деталей с различной толщиной опорной поверхности буксового проема, изнашиваемой в процессе эксплуатации. Эти испытания позволили разделить детали по степени напряженно-деформированного состояния и динамике изменения значимых параметров сигналов для различных источников АЭ. В результате исследований были выбраны значения пороговых параметров для всех типов зон контроля (табл. 2).

Способ был опробован на нескольких десятках боковых рам и надрессорных балок грузовых вагонов. Каждую деталь нагружали двумя видами нагрузки: продольное растяжение и трехточечный изгиб для боковых рам и трехточечный изгиб в направлении вверх и вниз для надрессорных балок. При диагностировании деталей о их состоянии судили по источнику с наибольшим определенным значением энергетического критерия. 
Таблица 2 - Критерии оценки состояния боковых рам и надрессорных балок для дискретного способа оценки устанавливаемого продленного пробега

\begin{tabular}{|c|c|c|c|c|}
\hline Зона & $E_{\min }$, Дж & $E_{\max }$, Дж & $Q_{\min }$, Дж & $Q_{\max }$, Дж \\
\hline 1 & 0,01 & 0,03 & 0,01 & 0,02 \\
\hline 2 & 0,03 & 0,1 & 0,02 & 0,04 \\
\hline 3 & 0,05 & 0,15 & 0,03 & 0,06 \\
\hline 4 & 0,1 & 0,3 & 0,05 & 0,1 \\
\hline
\end{tabular}

При испытаниях такого способа продления ресурса деталей использовали детали с различными дефектными состояниями. Были отобраны детали четырех категорий:

1) имеющие трещины различной длины и происхождения (например усталостные, горячие);

2) имеющие литейные объемные дефекты (раковины, выходящие на поверхность, пористости, неслитины, утяжины);

3) имеющие износы в ответственных узлах, увеличивающие напряжения на этих участках;

4) бездефектные детали (в которых визуальным и магнитными методами дефекты не были обнаружены).

В табл. 3 приведены обобщенные результаты диагностирования некоторых литых деталей, общее количество которых 50. В табл. 4 и 5 данные соответственно боковых рам и надрессорных балок, в которых присутствуют значимые локализованные источники, имеющие более одного сигнала из области нахождения дефекта.

Таблица 3 - Общие результаты представленных к диагностированию литых деталей тележки грузовых вагонов

\begin{tabular}{|c|c|c|c|c|}
\hline \multirow{2}{*}{$\begin{array}{c}\text { Группы } \\
\text { деталей }\end{array}$} & \multicolumn{2}{|c|}{ Боковые рамы } & \multicolumn{2}{c|}{ Надрессорные балки } \\
\cline { 2 - 5 } & Всего, шт. & $\begin{array}{c}\text { Идентифицированные } \\
\text { дефекты, шт. }\end{array}$ & Всего, шт. & $\begin{array}{c}\text { Идентифицированные } \\
\text { дефекты, шт. }\end{array}$ \\
\hline$a$ & 8 & 7 & 3 & 3 \\
\hline$\sigma$ & 5 & 3 & 2 & 0 \\
\hline 6 & 16 & 2 & 3 & 1 \\
\hline 2 & 11 & 0 & 12 & 2 \\
\hline
\end{tabular}

Как видно из общих данных, почти все трещины были активными источниками. Только одна трещина не являлась развивающейся и не излучала АЭ. Дальнейшее ее исследование с вырезкой и доломом образца, содержащего эту трещину, показало, что она была горячей и не развивалась вообще. В остальных случаях (б, в и г) только некоторые детали имели активные источники АЭ, которые можно было классифицировать по данной методике. Из годных по результатам других методов контроля деталей только одна надрессорная балка была забракована.

После испытаний детали, допущенные к дальнейшей эксплуатации, были использованы в течение назначенного по результатам прогнозирования срока в замкнутом маршруте.

После опытной эксплуатации была проведена дополнительная АЭ диагностика, которая показала, что незначительные и малозначительные источники в дальнейшем не были проявлены в результатах АЭ и детали были вновь отданы в эксплуатацию.

У боковой рамы № 846, имеющей единственный значительный дефект (панель локализации источника системой АЭ представлена на рис. 5), при повторной диагностике источник вновь излучал сигналы АЭ и деталь была выведена из дальнейшей эксплуатации. 
Таблица 4 - Результаты ресурсных АЭ испытаний боковых рам с локализованными активными источниками

\begin{tabular}{|c|c|c|c|c|c|c|}
\hline $\begin{array}{c}\text { № } \\
\text { детали }\end{array}$ & $\begin{array}{l}\text { Участок } \\
\text { детали }\end{array}$ & $N_{x}$ & $E_{\Sigma k}$, Дж & $\begin{array}{l}Q_{i j}, \\
\text { Дж }\end{array}$ & $\begin{array}{c}\text { Вид } \\
\text { дефекта }\end{array}$ & $\begin{array}{l}\text { Назначенный } \\
\text { ресурс от мак- } \\
\text { симального, \% }\end{array}$ \\
\hline 25425 & $\begin{array}{c}\text { Бок. плоскость } \\
\text { техн. окна }\end{array}$ & 10 & 1,674 & 0,714 & Критический & 0 \\
\hline 2670 & $\begin{array}{c}\text { Верхн. угол техн. } \\
\text { окна }\end{array}$ & 5 & 0,583 & 0,014 & Незначительный & 75 \\
\hline 16422 & $\begin{array}{c}\text { Нижний угол } \\
\text { техн. окна }\end{array}$ & 4 & 2,913 & 0,761 & Критический & 0 \\
\hline 62997 & $\begin{array}{c}\text { Внутр. угол букс. } \\
\text { проема }\end{array}$ & 8 & 0,899 & 0,379 & $-"-$ & 0 \\
\hline 15534 & $\begin{array}{c}\text { Верхн. угол техн. } \\
\text { окна }\end{array}$ & 5 & 1,799 & 0,745 & $-"-$ & 0 \\
\hline 21347 & $\begin{array}{c}\text { Верхн. плоск. } \\
\text { техн. окна }\end{array}$ & 2 & 0,430 & 0,012 & Незначительный & 75 \\
\hline 15885 & $\begin{array}{c}\text { Боковая плоск. } \\
\text { техн. окна }\end{array}$ & 8 & 0,697 & 0,299 & Критический & 0 \\
\hline 62884 & $\begin{array}{c}\text { Внутр. угол букс. } \\
\text { проема }\end{array}$ & 4 & 0,006 & 0,004 & Незначительный & 75 \\
\hline 10088 & $\begin{array}{l}\text { Наружн. угол } \\
\text { букс. проема }\end{array}$ & 5 & 0,779 & 0,278 & Критический & 0 \\
\hline 12979 & $\begin{array}{c}\text { Внутр. угол букс. } \\
\text { проема }\end{array}$ & 2 & 0,001 & 0,001 & Незначительный & 75 \\
\hline 846 & $\begin{array}{c}\text { Внутр. угол букс. } \\
\text { проема }\end{array}$ & 6 & 0,050 & 0,014 & $-"{ }_{-}$ & 75 \\
\hline
\end{tabular}

Таблица 5 - Результаты ресурсных АЭ испытаний надрессорных балок с локализованными активными источниками

\begin{tabular}{|c|c|c|c|c|c|c|}
\hline $\begin{array}{c}\text { № } \\
\text { детали }\end{array}$ & $\begin{array}{c}\text { Участок } \\
\text { детали }\end{array}$ & $N_{x}$ & $E_{\Sigma k}$, Дж & $Q_{i j}$ Дж & $\begin{array}{c}\text { Вид } \\
\text { дефекта }\end{array}$ & $\begin{array}{c}\text { Назначенный } \\
\text { ресурс от мак- } \\
\text { симального, \% }\end{array}$ \\
\hline 58634 & $\begin{array}{c}\text { Наклонная плос- } \\
\text { кость }\end{array}$ & 3 & 2,61 & 0,004 & $\begin{array}{c}\text { Малозначи- } \\
\text { тельный }\end{array}$ & 75 \\
\hline 45452 & $-{ }^{\prime \prime}-$ & 34 & 1,84 & 0,09 & $\begin{array}{c}\text { Критиче- } \\
\text { ский }\end{array}$ & 75 \\
\hline 6303 & $-{ }^{\prime \prime}-$ & 5 & 0,13 & 0,008 & $\begin{array}{c}\text { Малозначи- } \\
\text { тельный }\end{array}$ & 75 \\
\hline 7139 & $\begin{array}{c}\text { Опорная } \\
\text { повхость под- }\end{array}$ & 8 & 0,081 & 0,011 & $\begin{array}{c}\text { Незначи- } \\
\text { тельный }\end{array}$ & 100 \\
\hline 9942 & Нижний пояс & 12 & 0,026 & 0,012 & $\begin{array}{c}\text { Значитель- } \\
\text { ный }\end{array}$ & 25 \\
\hline 49452 & $\begin{array}{c}\text { Наклонная плос- } \\
\text { кость }\end{array}$ & 6 & 0,064 & 0,017 & $\begin{array}{c}\text { Незначи- } \\
\text { тельный }\end{array}$ & 100 \\
\hline
\end{tabular}




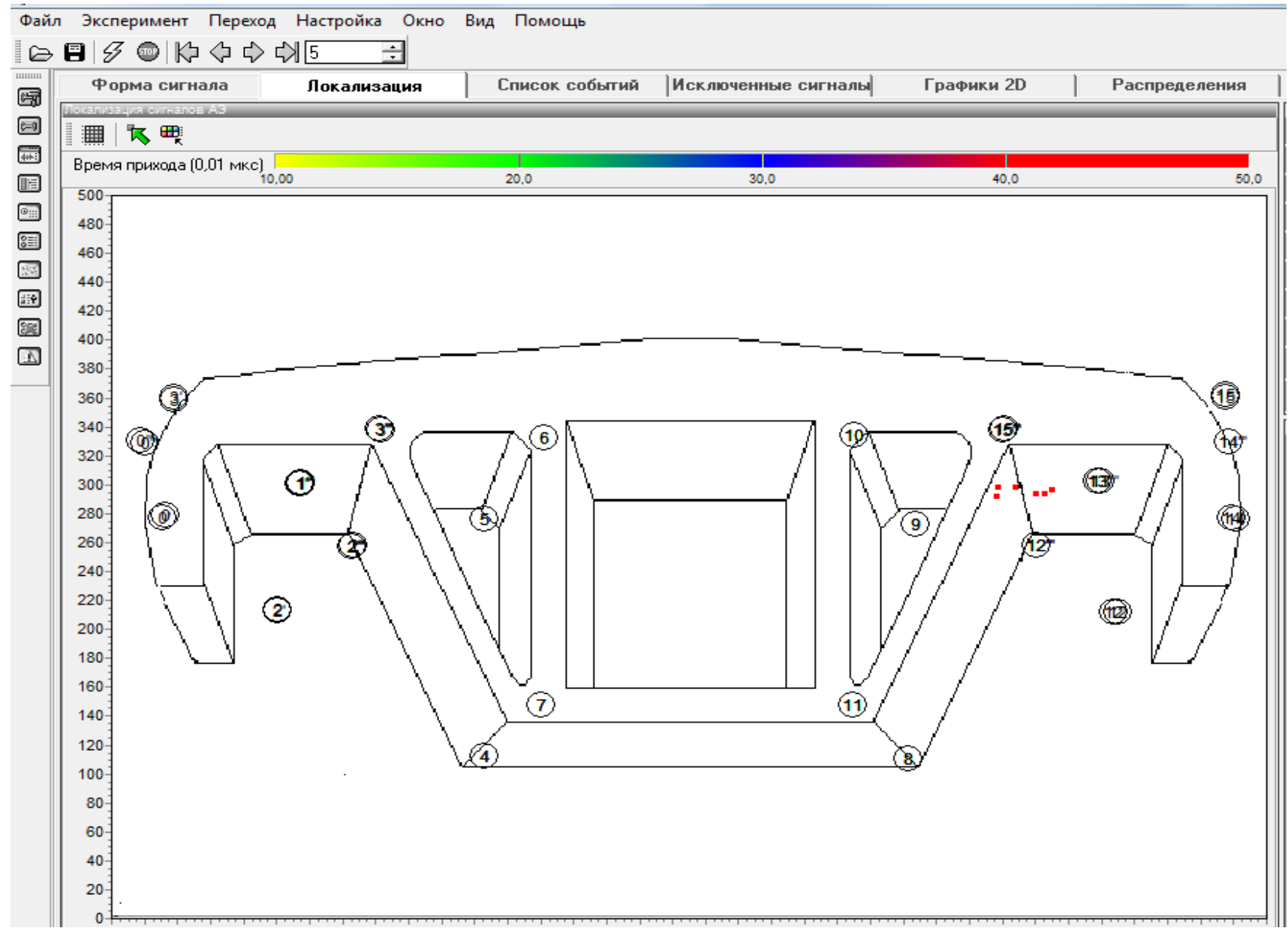

Рис. 5. Локализация дефекта во внутреннем углу буксового проема боковой рамы № 846

В целом можно отметить, что грань между малозначительными и значительными источниками наиболее тонкая и дальнейшее применение этого критерия для литых деталей показало, что детали со значительными дефектами, определенными по данной методике, встречаются реже всего. Также последующий набор результатов диагностирования литых деталей показывает, что надрессорные балки имеют активные источники примерно в два раза чаще, чем боковые рамы.

\section{Выводы}

Объекты сложной формы и сложного напряженного состояния обладают неравномерным напряженным состоянием в ходе эксплуатации, что позволяет расчетными и экспериментальными методами выделить наиболее потенциально опасные участки и выстраивать систему эффективного контроля. Примененные статистические методы практически позволили определить интервалы периодичности эксплуатационного контроля и определить эффективность контроля.

Разработана методика АЭ контроля с определением интегральных критериев оценки источников АЭ и определения параметров ресурса литых деталей сложной формы.

Такой подход удачно сочетается с моделью технологической диагностики в межремонтные периоды боковых рам и надрессорных балок на стационарных вагоноремонтных предприятиях. 
Методика позволяет ее использовать как самостоятельно, так и совместно с другими применяемыми методиками НК для оценки состояния боковых рам и надрессорных балок тележек грузовых вагонов в условиях вагоноремонтных предприятий.

\section{Литература}

1. Смирнов А. Н., Герике Б. Л., Муравьев В. В. Диагностирование технических устройств опасных производственных объектов. - Новосибирск : Наука, 2003. - 244 с.

2. Коллакот Р. Диагностика повреждений. - М. : Мир, 1989. - 516 с.

3. Иванов В. И. Оценка дополнительного времени эксплуатации объектов с применением акустико-эмиссионного контроля // Дефектоскопия. - 1982. - № 11. - С. 60-64.

4. Способ прогнозирования остаточного ресурса металлических изделий : пат. 2361199 Россйская Федерация / Бобров А. Л. ; опубл. 10.07.2009, Бюл. № 19. - 11 с.

5. Способ прогнозирования остаточного ресурса металлических изделий и устройство для его осуществления: пат. 2448343 Российская Федерация / Бобров А. Л., Степанова Л. Н., Кабанов С. И., Лебедев Е. Ю. ; опубл. 20.04.2012, Бюл. № 11. - 13 с.

6. Абрамов О. В., Градов О. М. Идентификация некоторых процессов эволюции дефектной структуры материала с источниками спонтанных акустических сигналов // Контроль. Диагностика. - 2000. - № 6. - С. 15-21.

7. Способ прогноза остаточного ресурса стальных металлоконструкций: пат. 2193772 Рос. Федерация / Берман А. В., Берман Д. В., Артеменко А. И., Шахмейстер Ю. Л., Воронцова И. А., Берман О. А., Штейнцайг Р. М., Хаспеков П. Р. - № 2001105893/28 ; заявл. 05.03.01 ; опубл. 27.11.02, Бюл. № 34. -8 с.

8. Robsman V. A. Nonlinear transformation of acoustic emission probability distributions with the evolution of the defect ensemble in a solid // Acoustical Physics. - 1996. - Vol. 42, no. 6. P. 749-754.

9. Исследования основных параметров сигналов акустической эмиссии при статических и циклических испытаниях образцов из стали 20ГЛ / Л. Н. Степанова, А. Л. Бобров, К. В. Канифадин, В. В. Чернова // Деформация и разрушение. - 2014. - № 6. - С. 41-47.

10. Недосека А. Я., Недосека С. А. Акустическая эмиссия и ресурс конструкций // Техническая диагностика и неразрушающий контроль. - 2008. - № 2. - С. 5-19.

11. Акустико-эмиссионный контроль боковой рамы тележки грузового вагона / С. А. Грассман, А. А. Больчанов, Л. Н. Степанова, С.А. Бехер, А.Л. Бобров, С.И. Кабанов // Железнодорожный транспорт. - 2011. - № 12. - С. 45-47.

12. Acoustic emission testing of solebars with boxlike cross section / L. N. Stepanova, S. A. Grassmann, A. L. Bobrov, S. A. Bekher, A. A. Bolchanov // Russian Journal of Nondestructive testing. - 2011. - Vol. 47, iss. 3. - P. 158-163. - DOI: 10.1134/S1061830911030090.

13. ПБ 03-593-03 Правила организации и проведения акустико-эмиссионного контроля сосудов, аппаратов, котлов и технологических трубопроводов. - М. : ГУП «Научно-технический центр по безопасности и промышленности Госгортехнадзор России», 2003. $-56 \mathrm{c}$.

14. Bobrov A. L. Analysys of Variations of the Dynamic Characteristics of Acoustic Emission Sources under Static Loading of Metal Speciemens // Russian Journal of Nondestructive Testing. 2009. - Vol. 45, iss. 5. - P. 304-309. - DOI: 10.1134/S1061830909050027. 